\title{
Serum Procalcitonin as a Biomarker to Determine the Duration of Antibiotic Therapy in Adult Patients with Sepsis and Septic Shock in Intensive Care Units: A Prospective Study
}

\author{
Gowri Vishalashi $\mathrm{SM}^{1} \oplus$, Poonam Gupta ${ }^{2}{ }^{\oplus}$, Pardeep Kumar Verma $\circledast^{3}$
}

\begin{abstract}
Background: Procalcitonin, a biomarker to adjudge the duration of antibiotic therapy in patients with sepsis.

Materials and methods: A prospective, randomized, controlled, interventional, single-center study was conducted in a mixed adult intensive care unit (ICU). In a nonblinded study, 90 adult patients admitted to the ICU with sepsis and septic shock were randomized into group P (group procalcitonin) and group C (group control). The duration of antibiotic therapy was decided based on serum procalcitonin levels for patients in group P versus standard treatment protocols in group C. A procalcitonin value of $<0.01 \mathrm{ng} / \mathrm{mL}$ or a subsequent decline of $>80 \%$ from the baseline was cutoff and chosen to stop the antibiotic therapy. The primary aim was to compare the duration of antibiotic therapy (in days) in the two groups. The secondary objective was to compare and assess the length of ICU stay, reinfection, secondary infection rate, readmission rate, and mortality among the groups.

Results: The mean duration of antibiotic therapy was significantly lesser in patients in group $\mathrm{P}(4.98 \pm 2.56$ vs $7.73 \pm 3.06$ days, $p<0.001)$. Patients in group C spent more days in ICU $(8.80 \pm 3.35$ vs $5.98 \pm 2.73$ days, $p<0.001)$. The secondary infection rate was significantly higher in group C ( $26.7 \%$ vs $4.4 \%, p=0.014)$. Readmission and mortality rates were comparable between the groups.

Conclusion: Serum procalcitonin-based algorithm in critically ill patients with sepsis could lead to a reduction in the duration of antibiotic therapy, ICU stay, and associated morbidities like secondary infection rates. It further promotes antibiotic stewardship without any adverse effects on the patient's outcome.

Keywords: Antibiotic stewardship, Antibiotic therapy, Antimicrobial resistance, Biomarkers, Procalcitonin, Sepsis.

Indian Journal of Critical Care Medicine (2021): 10.5005/jp-journals-10071-23802
\end{abstract}

\section{INTRODUCTION}

Antimicrobial resistance is alarmingly increasing owing to the unwarranted, inappropriate, and prolonged use of antibiotics. Antimicrobial resistance not only provoking a major threat to global health but also precipitating a huge negative impact on the fields of medicine that are highly dependent on the availability of antimicrobial drugs. ${ }^{1-4}$ Sepsis accounts for more than half of ICU admissions with mortality ranging from $30 \%$ to $50 \% .{ }^{5}$ Early institution of antibiotics and timely de-escalation are important considerations in the management of sepsis. ${ }^{6}$ Several biomarkers have been described for severity assessment of infection and also help in the de-escalation of antibiotics. ${ }^{7}$ Various randomized controlled trials, on patients with sepsis, have proved that a treatment algorithm based on serum procalcitonin is a promising way for guiding antibiotic therapy with high sensitivity and without any undesirable effects on the outcome. . $^{8,9}$

Thus, the present study was conducted to assess the role of serum procalcitonin levels in determining the duration of antibiotics in critically ill patients with sepsis and septic shock in ICU. The primary objective was to compare the duration of antibiotic therapy (in days) when guided by serial serum procalcitonin levels with that of the standard institutional protocol. The secondary objectives were to compare the length of ICU stay, reinfection rates, and mortality among the two groups.
${ }^{1-3}$ Department of Anaesthesia and Intensive Care, Safdarjung Hospital and Vardhman Mahavir Medical College, New Delhi, India

Corresponding Author: Pardeep Kumar Verma, Department of Anaesthesia and Intensive Care, Safdarjung Hospital and Vardhman Mahavir Medical College, New Delhi, India, Phone: +91 9818402209, e-mail: prateekverma88@hotmail.com

How to cite this article: Vishalashi SMG, Gupta P, Verma PK. Serum Procalcitonin as a Biomarker to Determine the Duration of Antibiotic Therapy in Adult Patients with Sepsis and Septic Shock in Intensive Care Units: A Prospective Study. Indian J Crit Care Med 2021;25(5):507511.

Source of support: Nil

Conflict of interest: None

\section{Materials and Methods}

\section{Study Design and Setting and Research Population}

This prospective, randomized, controlled, interventional study was conducted in the mixed adult ICUs of the tertiary hospital over a period of 18 months from September 2018 to February 2020. In this nonblinded study, after obtaining Institute Ethical Committee approval, adult patients with sepsis or septic shock based on sepsis 3 criteria who were admitted to ICUs within 24 hours were included. Patients who did not consent to participate in the study,

() Jaypee Brothers Medical Publishers. 2021 Open Access This article is distributed under the terms of the Creative Commons Attribution 4.0 International License (https://creativecommons.org/licenses/by-nc/4.0/), which permits unrestricted use, distribution, and non-commercial reproduction in any medium, provided you give appropriate credit to the original author(s) and the source, provide a link to the Creative Commons license, and indicate if changes were made. The Creative Commons Public Domain Dedication waiver (http://creativecommons.org/publicdomain/zero/1.0/) applies to the data made available in this article, unless otherwise stated. 
pregnant patients, when prolonged antibiotic therapy is indicated (e.g., infective endocarditis), viral or parasitic infections, severely immunocompromised patients, patients who were admitted merely for postoperative observation, and an estimated length of stay in ICU less than a day were excluded from the study.

Sample size was calculated based on the study by Nobre et al. ${ }^{10}$ The approximate duration of antibiotic therapy was 9.5 days and 6 days in group $C$ and group $P$, respectively, in the study ${ }^{10}$, and the mean difference was 3.2 days. With these values as the reference and assuming a standard deviation of 4.5 with $90 \%$ power of the study and $5 \%$ level of significance, a sample size of 45 patients per study group was calculated. The estimated percentage reduction in the duration of antibiotic therapy in group P was $36.8 \%$.

Ninety patients were randomized into two groups based on block randomization using a sealed envelope technique as follows:

- Group P $(n=45)$ : procalcitonin group. \{Interventional Arm\}

- Group C ( $n=45)$ : control group. \{Comparator Arm\}

At admission, patients in both groups were assessed clinically and thereafter continuously during their ICU stay. APACHE II and SOFA scores were calculated for patients in both the groups on admission and then on alternate days. Routine blood investigations such as complete blood count, coagulation profile, kidney and liver function tests, etc. were done at the time of admission and then on alternate days. Appropriate samples were taken for microbiological culture and sensitivity, at the time of admission before the initiation of antibiotics and then once in two days. Arterial blood gas analysis and serum lactate levels were done daily and whenever indicated. Twelve lead ECG and chest X-ray were done initially and whenever indicated. Any other investigation if required was done, as and when indicated. All the above-mentioned investigations and other special investigations were done as and when indicated for patients in both groups.

Serial serum procalcitonin measurements were done for patients in both groups.

Serum procalcitonin values were measured using VIDAS ${ }^{\circledR}$ B.R.A.H.M.S. $\mathrm{PCT}^{\mathrm{TM}}$, an automated test for the determination of procalcitonin levels in human serum or plasma. Patients in both groups received standard treatment as per institutional protocol.

For patients in group C, antibiotics were given as per institutional protocol despite the change in serum procalcitonin levels. The institutional protocol is to administer antibiotic therapy for 7 days for the lower respiratory tract, urinary tract, and wound site bacterial infection and 10 days for bloodstream infection.

For patients in group $\mathrm{P}$, the duration of antibiotic therapy was decided based on the procalcitonin algorithm.

Antibiotic therapy was stopped if

1. procalcitonin $<0.10 \mathrm{ng} / \mathrm{mL}$ or

2. Subsequent decline in procalcitonin $>80 \%$ from the baseline.

In both groups, the clinical status of the patient was also considered before stopping the antibiotic therapy.

\section{Statistical Analysis}

Observational data were analysed using SPSS version 21.0. Categorical variables were presented in number and percentage (\%), and continuous variables were presented as mean \pm SD and median. Normality of data was tested by Kolmogorov-Smirnov test. Nonparametric tests were used in case normality was rejected. Quantitative variables were compared using unpaired t-test or Mann-Whitney test (when the data sets were not normally distributed) between the groups. Qualitative variables were compared using chi-square or Fisher's exact test. A $p$ value of $<0.05$ was considered statistically significant.

\section{Result}

The demographic profile and baseline parameters like serum procalcitonin, APACHE II and SOFA scores, and serum lactate levels were comparable between groups $C$ and $P$. No significant difference was noted in the distribution of the type of patient, i.e., medical versus surgical patients between the two groups (Table 1). The number of patients with septic shock was higher in group $C$ patients than that in group P patients, and the difference was statistically significant $(p=0.006)$. The number of patients with bacteremia on day $1(p=0.0516)$ and in whom a multidrug-resistant organism was isolated in the initial culture $(p=0.4795)$ were comparable between the groups. The mean serum procalcitonin levels were also comparable at all time intervals (Fig. 1). Eleven organisms were

Table 1: Demographic parameters and baseline investigations

\begin{tabular}{llll}
\hline Parameter & Group C & Group P & p value \\
\hline $\begin{array}{l}\text { Age (years) } \\
\text { Gender: male/ }\end{array}$ & $46.78 \pm 16.22$ & $42.89 \pm 16.83$ & 0.267 \\
female (numbers) & $29 / 16$ & $22 / 23$ & 0.136 \\
$\begin{array}{l}\text { Speciality: medical/ } \\
\text { surgical (\%) }\end{array}$ & $42.2 / 57.8$ & $40 / 60$ & 0.830 \\
$\begin{array}{l}\text { Temperature ( }{ }^{\circ} \text { F) } \\
\text { (mean } \pm \text { SD) }\end{array}$ & $98.93 \pm 1.84$ & $99.5 \pm 2.29$ & 0.195 \\
$\begin{array}{l}\text { TLC (cells/mm }{ }^{3} \text { ) } \\
\text { Septic shock (\%) }\end{array}$ & $16,100 \pm 3362.94$ & $15,500 \pm 4501.87$ & 0.535 \\
$\begin{array}{l}\text { Bacteremia on } \\
\text { day } 1 \text { (\%) }\end{array}$ & 28.2 & 55.6 & 0.006 \\
$\begin{array}{l}\text { Serum lactate } \\
\text { level (mmol/L) } \\
\text { (mean } \pm \text { SD) }\end{array}$ & $3.08 \pm 1.41$ & $3.02 \pm 1.42$ & 0.858 \\
$\begin{array}{l}\text { Serum procal- } \\
\text { citonin (ng/mL) } \\
\text { (mean } \pm \text { SD) }\end{array}$ & $8.88 \pm 20.63$ & $8.88 \pm 18.87$ & 1.000 \\
$\begin{array}{l}\text { APACHE II score } \\
\text { (mean } \pm \text { SD) }\end{array}$ & $17.4 \pm 5.53$ & $18.07 \pm 6.67$ & 0.607 \\
$\begin{array}{l}\text { SOFA score } \\
\text { (mean } \pm \text { SD) }\end{array}$ & $4.8 \pm 2.47$ & $4.18 \pm 3.45$ & 0.328 \\
$\begin{array}{l}\text { MDR organism in } \\
\text { initial culture (\%) }\end{array}$ & 11.11 & 6.66 & 0.479 \\
\hline
\end{tabular}

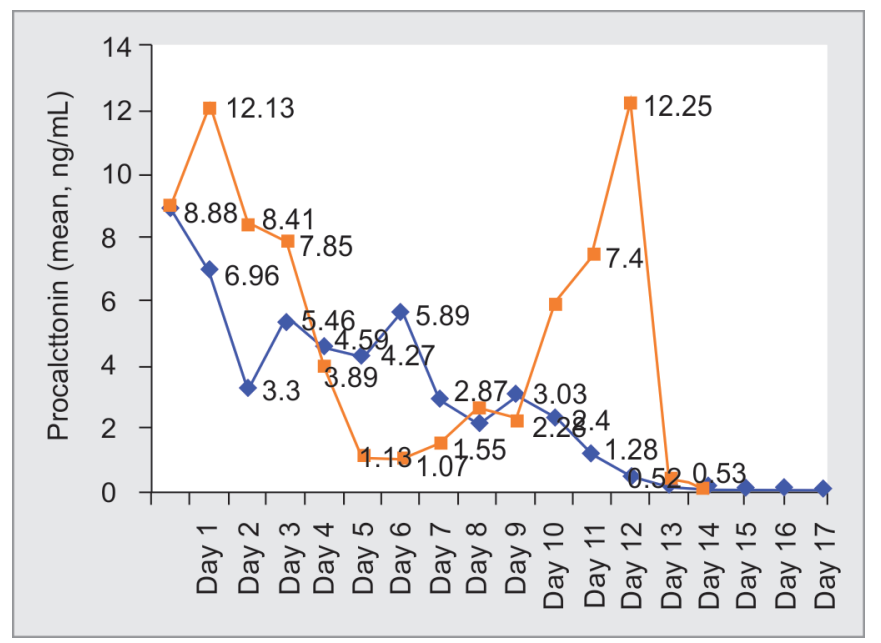

Fig. 1: Procalcitonin trends in groups $C$ and $P$ 


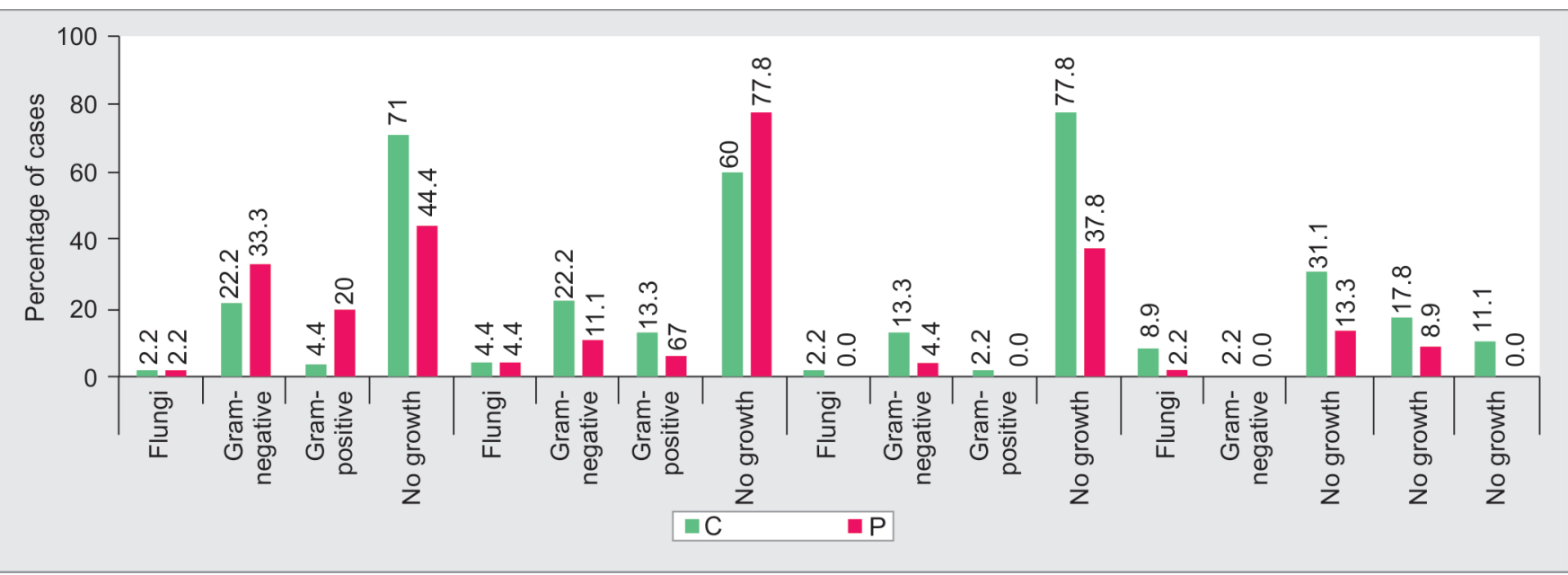

Fig. 2: Depicting microbiological cultures between groups $C$ and $P$

Table 2: Additional outcome measures

\begin{tabular}{llll}
\hline Parameter & Group C & Group P & p value \\
\hline $\begin{array}{l}\text { Duration of mechanical } \\
\text { ventilation (days) } \\
\text { (mean } \pm \text { SD) }\end{array}$ & $3.18 \pm 2.30$ & $2.00 \pm 2.30$ & 0.022 \\
$\begin{array}{l}\text { Duration of inotrope } \\
\text { requirement (days) } \\
\text { (mean } \pm \text { SD) }\end{array}$ & $5.87 \pm 2.94$ & $4.49 \pm 2.12$ & 0.013 \\
Need for dialysis, no. (\%) & $7(15.6 \%)$ & $3(6.7 \%)$ & 0.315 \\
\hline
\end{tabular}

Table 3: Primary and secondary outcome measures

\begin{tabular}{llll}
\hline Parameter & Group C & Group P & p value \\
\hline $\begin{array}{l}\text { Duration of antibiotic } \\
\text { therapy (days) (mean } \pm \text { SD) }\end{array}$ & $7.73 \pm 3.06$ & $4.98 \pm 2.56$ & $<0.001$ \\
Reinfection & $4.4 \%$ & $6.7 \%$ & 0.635 \\
$\begin{array}{l}\text { Secondary infection } \\
\text { Duration of ICU stay (days) }\end{array}$ & $26.7 \%$ & $4.4 \%$ & 0.014 \\
(mean \pm SD) & $8.80 \pm 3.35$ & $5.98 \pm 2.73$ & $<0.001$ \\
$\begin{array}{l}\text { Readmission in ICU for } 28 \\
\text { days, no. (\%) }\end{array}$ & $6(13.3 \%)$ & $4(8.9 \%)$ & 0.739 \\
ICU mortality, no. (\%) & $7(15.6)$ & $4(8.9 \%)$ & 0.522 \\
\hline
\end{tabular}

isolated from various cultures. Gram-positive and gram-negative infections were initially higher in group $\mathrm{P}$ compared to group $\mathrm{C}$, but later, gram-positive, gram-negative, and fungal infections predominated and were significantly higher in group $C$ than that in group $\mathrm{P}$ ( $p<0.001$ for day $6 ; p=0.023$ for day 9 ) (Fig. 2). The mean duration of antibiotic therapy (7.73 vs 4.98 days; $p<0.001$ ), secondary infection rate $(26.7 \%$ vs $4.4 \% ; p=0.014)$, the average duration of a patient on mechanical ventilation (3.18 vs 2 days; $p=0.022$ ), and the mean duration of ICU stay (8.8 vs 5.98 days; $p<0.001$ ) were higher in group C (Tables 2 and 3). Patients in group $C$ had a $15.6 \%$ mortality, and patients in group $\mathrm{P}$ had $8.9 \%$ mortality in the ICU; but the difference was not significant $(p=0.522)$ (Table 3 ).

\section{Discussion}

In critically ill patients, early initiation of antibiotics is the foremost recommendation in surviving sepsis guidelines, but overt and improper use is resulting in multiresistant strains. The role of procalcitonin in antibiotic stewardship in critically ill patients was studied in this prospective study.

In this study, patients with the both medical and surgical causes of sepsis were included, and it was comparable in both groups (group $C$ and group P). In SAPS, ProGUARD, and PRORATA trials, the majority of patients were of the pulmonary origin of sepsis. ${ }^{1,10-12}$

In our study, patients with septic shock were predominated in group C (82.2\%) than in group P (55.6\%) similar to the MOSES study. ${ }^{13}$ In contrast, Nobre et al. had more number of patients with severe sepsis than the number of patients with only sepsis. ${ }^{10}$ More patients with septic shock in group $C$ could be the reason for a higher a qSOFA score in that group in our study, but the difference was not statistically significant.

The mean baseline procalcitonin levels were $8.88 \pm 20.63$ versus $8.88 \pm 18.87 \mathrm{ng} / \mathrm{mL}$ (mean $\pm \mathrm{SD}$ ) in groups $C$ and $P$, respectively, which was comparable and similar to the findings by Nobre et al. ${ }^{10}$ We observed a significant reduction in the duration of antibiotic therapy in group $\mathrm{P}(4.98 \pm 2.567$ vs $7.3 \pm 3.06, p<0.001)$. The mean difference observed in the duration of antibiotic therapy was 2.75 days $(p<0.001)$. Our study showed that the discontinuation of antibiotic therapy based on a procalcitonin algorithm regulated in reduced antibiotic duration similar to various past trials.,10,12

Similar to our study, Shehabi et al. and the ProGUARD study used a similar conservative cutoff of $0.1 \mu \mathrm{g} / \mathrm{L}$, but they did not find any significant reduction in the duration of antibiotic therapy in group $\mathrm{P}^{11}$ whereas most of the other major randomized trials used a higher procalcitonin cutoff to stop antibiotic therapy. Nobre et al., ${ }^{10}$ Stolz et al., ${ }^{14}$ and Boudama et al. ${ }^{15}$ used a procalcitonin cutoff of $0.25 \mu \mathrm{g} / \mathrm{L}$ or below in which the use of antibiotics was strongly discouraged. De Jong et al. used a procalcitonin cutoff of $0.5 \mu \mathrm{g} / \mathrm{L}$ or $a \geq 80 \%$ reduction from the peak procalcitonin values. ${ }^{1}$ All the above studies reported a significant reduction in the duration of antibiotic therapy following a procalcitonin-based algorithm. A conservative cutoff of $\leq 0.1 \mu \mathrm{g} / \mathrm{mL}$ was chosen to increase the safety margin, which stands justified because of the possibility of a lower baseline procalcitonin level despite positive cultures. 
Reinfection was considered when a particular organism was isolated at the same site once the cultures had become sterile with antibiotic therapy, whereas the secondary infection was considered when a second organism was isolated from any site while under treatment for a primary infection.

Secondary infection during ICU stay was significantly higher in group $C$ than that in group $\mathrm{P}(26.7 \%$ vs $4 \% ; p=0.014)$, while the reinfection rates were comparable between the groups in this study. This is in contrast to the observations by De Jong et al., who observed significantly higher reinfection rates in group $\mathrm{P}(p=0.0492){ }^{1}$ Nobre et al. ${ }^{10}$ and Boudama et al. ${ }^{15}$ observed no difference in reinfection rates between the groups similar to our study.

The duration of ICU stay was significantly lower in group $P$ with a mean difference of 2.82 days $(p<0.001)$; in contrast to the results of the PRORATA, ${ }^{15}$ ProGUARD ${ }^{7}$, and SAPS trials, ${ }^{1}$ no significant difference in the length of ICU stay between the groups was observed. However, Nobre et al. reported a significant reduction in the length of ICU stay (a reduction of ICU length of stay by 2 days; $p=0.03) .{ }^{10}$ Prolonged ICU stay of patients in group $C$ might be the reason for higher secondary infection rates.

Also, patients in group $C$ had spent a significantly longer duration on mechanical ventilation compared to group $P$ (mean difference in duration of mechanical ventilation 1.18 days; $p=0.022$ ) and were on inotropic support for a significantly longer duration ( 5.87 vs 4.45 days, respectively; $p=0.013$ ). These findings attributed to the fact that more patients in group $C$ had septic shock compared to group P. The number of patients who landed up in acute kidney injury needing dialysis was comparable between the groups. In contrast, Jensen et al. in procalcitonin and survival study observed that the patients in whom the procalcitonin-based algorithm was used for antibiotic escalation had spent a long time needing dialysis than the patients in group $C .{ }^{16}$ We did not observe any significant difference in the ICU mortality between the groups \{group C $15.6 \%$ vs group P $8.9 \% ; p=0.52\}$ similar to Bloos et al. ${ }^{17}$ and Shehabi et al. ${ }^{11}$

Similar to our observations, Townsend et al. did not find any significant difference in the all-cause hospital readmission within 30 days between the groups $(p=0.36) .^{17}$

\section{LiMITATIONS}

This was the single-center study with a smaller sample size. The study design was not blinded, and the possibility of bias cannot be excluded. The likelihood of a patient having received an antibiotic before admission to the ICU and its effects on procalcitonin levels was not taken into consideration. Comparatively, a poorer outcome in group $C$ can be attributed to fact that the number of patients with septic shock was significantly higher in group C compared to group P. Patients who need antibiotic therapy for a prolonged duration, patients with viral and parasitic infections, pregnant patients, and immunocompromised patients were excluded from the study. Thus, generalizing the findings to a population that would contain all the above-mentioned patients is not possible. Further multicenter trials enrolling larger populations are needed to validate the results of our study.

\section{Conclusion}

Serum procalcitonin-based antibiotic prescription guideline with a low cutoff of $0.1 \mu \mathrm{g} / \mathrm{mL}$ can be used to guide the duration of antibiotic therapy with a significant reduction in the duration of antibiotic therapy and morbidity, in terms of duration of ICU stay, secondary infection, and days spent on mechanical ventilation.

\section{ORCID}

Gowri Vishalashi SM ๑ https://orcid.org/0000-0002-5556-1433

Poonam Gupta ๑ https://orcid.org/0000-0002-3075-3412

Pardeep Kumar Verma @ https://orcid.org/0000-0003-4120-1266

\section{References}

1. De Jong $E$, van Oers J, Beishuizen A, Vos P, Vermeijden W, Haas L, et al. Efficacy and safety of procalcitonin guidance in reducing the duration of antibiotic treatment in critically ill patients: a randomised, controlled, open-label trial. Lancet Infect Dis 2016;16:819-827. DOI: 10.1016/S1473-3099(16)00053-0.

2. Schuetz $P$, Christ-Crain M, Thomann R, Falconnier C, Wolbers M, Widmer I, et al. Effect of procalcitonin-based guidelines vs standard guidelines on antibiotic use in lower respiratory tract infections: the ProHOSP randomized controlled trial. JAMA 2009;302:1059-1066. DOI: 10.1001/jama.2009.1297.

3. Prestinaci F, Pezzotti P, Pantosti A. Antimicrobial resistance: a global multifaceted phenomenon. Pathog Glob Health 2015;109:309-318. DOI: 10.1179/2047773215Y.0000000030.

4. Huttner A, Harbarth S, Carlet J, Cosgrove S, Goossens H, Holmes A, et al. Antimicrobial resistance: a global view from the 2013 World Healthcare-Associated Infections Forum. Antimicrob Resist Infect Control 2013;2:31. DOI: 10.1186/2047-2994-2-31.

5. Rhee C, Jones TM, Hamad Y, Pande A, Varon J, O'Brien C, et al. Prevalence, underlying causes, and preventability of sepsisassociated mortality in US acute care hospitals. JAMA Netw Open 2019;2:e187571. DOI: 10.1001/jamanetworkopen.2018.7571.

6. Shorr AF, Micek ST, Welch EC, Doherty JA, Reichley RM, Kollef MH. Inappropriate antibiotic therapy in Gram-negative sepsis increases hospital length of stay. Crit Care Med 2011;39:46-51. DOI: 10.1097/ CCM.0b013e3181fa41a7.

7. Vincent JL, Teixeira L. Sepsis biomarkers. Value and limitations. Am J Resp Crit Care Med 2014;190:1081-1082. DOI: 10.1164/rccm.2014101895ED.

8. Nargis W, Ibrahim M, Ahamed BU. Procalcitonin versus C-reactive protein: usefulness as biomarker of sepsis in ICU patient. Int J Crit Illn Inj Sci 2014;4:195-199. DOI: 10.4103/2229-5151.141356.

9. Müller B, Becker KL, Schächinger H, Rickenbacher PR, Huber PR, Zimmerli W, et al. Calcitonin precursors are reliable markers of sepsis in a medical intensive care unit. Crit Care Med 2000;28:977-983. DOI: 10.1097/00003246-200004000-00011.

10. Nobre V, Harbarth S, Graf J, Rohner P, Pugin J. Use of procalcitonin to shorten antibiotic treatment duration in septic patients. Am J Respir Crit Care Med 2008;177;498-505. DOI: 10.1164/rccm.200708-1238OC.

11. Shehabi Y, Sterba M, Garrett PM, Rachakonda KS, Stephens D, Harrigan $\mathrm{P}$, et al. Procalcitonin algorithm in critically ill adults with undifferentiated infection or suspected sepsis. A randomized controlled trial. Am J Respir Crit Care Med 2014;190:1102-1110. DOI: 10.1164/rccm.201408-14830C.

12. Boudama L, Luyt C, Tubach F, Cracco C, Alvarez A, Schwebel C, et al. Use of procalcitonin to reduce patients' exposure to antibiotics in intensive care units (PRORATA trial): a multicentre randomised controlled trial. Lancet 2010;375:463-474. DOI: 10.1016/S01406736(09)61879-1.

13. Schuetz P, Birkhahn R, Sherwin R, Jones A, Singer A, Kline J, et al. Serial procalcitonin predicts mortality in severe sepsis patients. Crit Care Med 2017;45:781-789. DOI: 10.1097/CCM.0000000000002321.

14. Stolz D, Smyrnios N, Eggimann P, Pargger H, Thakkar N, Siegemund $M$, et al. Procalcitonin for reduced antibiotic exposure in ventilatorassociated pneumonia: a randomised study. Eur Respir J 2009;34:13641375. DOI: $10.1183 / 09031936.00053209$.

15. Jensen JU, Hein L, Lundgren B, Bestle MH, Mohr TT, Andersen MH, et al. Procalcitonin-guided interventions against infections to increase early appropriate antibiotics and improve survival in the intensive care unit: a randomized trial. Crit Care Med 2011;39:20482058. DOI: 10.1097/CCM.0b013e31821e8791. 
16. Bloos F, Trips E, Nierhaus A, Briegel J, Heyland DK, Jaschinski U, et al. Effect of sodium selenite administration and procalcitononguided therapy on mortality in patients with severe sepsis or septic shock A randomized clinical trial. JAMA Intern Med 2016;176:1266-1276. DOI: 10.1001/jamainternmed.2016. 2514.
17. Townsend J, Adams V, Galiatsatos, Pearse D, Pantle H, Masterson $M$, et al. Procalcitonin-guided antibiotic therapy reduces antibiotic use for lower respiratory tract infections in a United States medical center: results of a clinical trial. Open Forum Infect Dis 2018;5:ofy327. DOI: 10.1093/ofid/ofy327. 